\title{
On the numerical solution of nonlinear fractional-integro differential equations
}

\author{
Mehmet Senol $^{1}$ and Hamed Daei Kasmaei ${ }^{2}$ \\ ${ }^{1}$ Department of Mathematics, Faculty of Science and Literature, Nevsehir Haci Bektas Veli University, Nevsehir, Turkey \\ ${ }^{2}$ Department of Mathematics and Statistics, Faculty of Science, Central Tehran Branch, Islamic Azad University, Tehran, Iran
}

Received: 5 December 2016, Accepted: 6 February 2017

Published online: 25 August 2017.

\begin{abstract}
In the present study, a numerical method, perturbation-iteration algorithm (shortly PIA), has been employed to give approximate solutions of some nonlinear Fredholm and Volterra type fractional-integro differential equations (FIDEs). Comparing with the exact solution, the PIA produces reliable and accurate results for FIDEs.
\end{abstract}

Keywords: Fractional-integro differential equations, Caputo fractional derivative, Initial value problems, Perturbation-Iteration Algorithm.

\section{Introduction}

Scientists has been interested in fractional order calculus as long as it has been in classical integer order analysis. However, for many years it could not find practical applications in physical sciences. Recently, fractional calculus has been used in applied mathematics, viscoelasticity [41], control [34], electrochemistry [31], electromagnetic [14].

Developments in symbolic computation capabilities is one of the driving forces behind this rise. Different multidisciplinary problems can be handled with fractional derivatives and integrals.

[25] and [33] are studies that describe the fundamentals of fractional calculus give some applications. Existence and uniqueness of the solutions are also studied in [40,38].

Similar to the studies in physical sciences, fractional order integro differential equations (FIDEs) also gave scientists the opportunity of describing and modeling many important and useful physical problems.

In this manner, a remarkable effort has been expended to propose numerical methods for solving FIDEs, in recent years. Fractional variational iteration method [19,20], homotopy analysis method [23,7], finite element method [11,12], fractional differential transform method [29,3,10] and Adams-Bashforth-Moulton Method [4,5] are among these methods.

The aim of this study was to construct and test an algorithm using PIA to obtain approximate solutions of some nonlinear fractional order Fredholm and Volterra type integro-differential equation. In the present study we also give the convergence analysis of the method for the first time. This method can be applied to a wide range of problems without requiring any special assumptions and restrictions. 
A few fractional derivative definitions of an arbitrary order exists in the literature. Two most used of them are the Riemann-Liouville and Caputo fractional derivatives. The two definitions are quite similar but have different order of evaluation of derivation.

Due to the appropriateness of the initial conditions, fractional definition of Caputo is often used in recent years.

\section{Basic definitions}

Definition 1. A real function $f(t), t>0$ is said to be in the space $C_{\mu},(\mu>0)$ if there exists a real number $p(>\mu)$, such that $f(t)=t^{p} f_{1}(t)$ where $f_{1} \in C[0, \infty)$, and it is said to be in the space $C_{\mu}^{m}$ if $f^{(m)} \in C_{\mu}, m \in \aleph[24]$.

Definition 2. The Riemann-Liouville fractional integral operator $\left(J^{\alpha}\right)$ of order $\alpha \geq 0$, of a function $f \in C_{\mu}, \mu \geq-1$ is defined as [25].

$$
J^{\alpha} f(t)=\frac{1}{\Gamma(\alpha)} \int_{0}^{t}(t-\tau)^{\alpha-1} f(\tau) d \tau, \quad \alpha, t>0
$$

and $J^{0} f(t)=f(t)$, where $\Gamma$ is the well-known gamma function. For $f \in C_{\mu}, \mu \geq-1, \alpha, \beta \geq 0$ and $\lambda>-1$, the following properties hold.

(i) $J^{\alpha} J^{\beta} f(t)=J^{\alpha+\beta} f(t)$,

(ii) $J^{\alpha} J^{\beta} f(t)=J^{\beta} J^{\alpha} f(t)$,

(iii) $J^{\alpha} t^{\lambda}=\frac{\Gamma(\lambda+1)}{\Gamma(\lambda+1+\alpha)} t^{\lambda+\alpha}$.

Definition 3. The Caputo fractional derivative of $f$ of order $\alpha, f \in C_{-1}^{m}, m \in \mathfrak{\aleph} \cup\{0\}$, is defined as [33].

$$
D^{\alpha} f(t)=J^{m-\alpha} f^{(m)}(t)=\frac{1}{\Gamma(m-\alpha)} \int_{0}^{t}(t-\tau)^{m-\alpha-1} f^{(m)}(\tau) d \tau, \quad \alpha, t>0,
$$

where $m-1<\alpha<m$ with the following properties;

(i) $D^{\alpha}(a f(t)+b g(t))=a D^{\alpha} f(t)+b D^{\alpha} g(t), a, b \in \mathfrak{R}$,

(ii) $D^{\alpha} J^{\alpha} f(t)=f(t)$,

(iii) $J^{\alpha} D^{\alpha} f(t)=f(t)-\sum_{j=0}^{k-1} f^{(j)}(0) \frac{t^{j}}{j !}, t>0$.

After this introductory section, section 3 is reserved to a brief review of the Perturbation-Iteration Algorithm PIA, in section 4 convergence analysis of the present method is given, in section 5 some examples are illustrated to show the simplicity and effectiveness of the algorithm. Finally the paper ends with a conclusion in section 6.

\section{Analysis of the PIA}

Differential equations are naturally used to describe problems in engineering and other applied sciences. Searching approximate solutions for complicated equations has always attracted attention. Many different methods and frameworks exist for this purpose and perturbation techniques [30,22,37] are among them. These techniques can be used to find approximate solutions for both ordinary and partial differential equations.

Requirement of a small parameter in the equation that is sometimes artificially inserted is a major limitation of perturbation techniques that renders them valid only in a limited range. Therefore, to overcome the disadvantages come with the perturbation techniques, several methods have been proposed by authors $[15,26,27,28,8,18,39,13,21,16]$. 
Parallel to these attempts, a perturbation-iteration method has been proposed by Aksoy, Pakdemirli and their co-workers $[1,32,2]$ previously. In the new technique, an iterative algorithm is constructed on the perturbation expansion. The present method has been tested on Bratu-type differential equations [1] and first order differential equations [32] with success. Then the algorithms were applied to nonlinear heat equations also [2]. The solutions of the Volterra and Fredholm type integral equations [9], first-order differential equations and systems [35] and solutions of ordinary frational differental equations [36] have been presented by the developed method, finally.

This new algorithm have not been used for any fractional integro differential equations yet. To obtain the approximate solutions of FIDEs, the most basic perturbation-iteration algorithm PIA(1,1) is employed by taking one correction term in the perturbation expansion and correction terms of only first derivatives in the Taylor series expansion. [1,32,2].

Take the fractional-integro differential equation.

$$
F\left(u^{(\alpha)}, u, \int_{0}^{t} g(t, s, u(s)) d s, \varepsilon\right)=0
$$

where $u=u(t)$ and $\varepsilon$ is a small parameter. The perturbation expansions with only one correction term is

$$
\begin{gathered}
u_{n+1}=u_{n}+\varepsilon\left(u_{c}\right)_{n}, \\
u_{n+1}^{\prime}=u_{n}^{\prime}+\varepsilon\left(u_{c}^{\prime}\right)_{n} .
\end{gathered}
$$

Replacing Eq.(8) into Eq.(7) and writing in the Taylor series expansion for only first order derivatives gives

$$
\begin{aligned}
& F\left(u_{n}^{(\alpha)}, u_{n}, \int_{0}^{t} g\left(t, s, u_{n}(s)\right) d s, 0\right)+F_{u}\left(u_{n}^{(\alpha)}, u_{n}, \int_{0}^{t} g\left(t, s, u_{n}(s)\right) d s, 0\right) \varepsilon\left(u_{c}\right)_{n} \\
& +F_{u^{(\alpha)}}\left(u_{n}^{(\alpha)}, u_{n}, \int_{0}^{t} g\left(t, s, u_{n}(s)\right) d s, 0\right) \varepsilon\left(u_{c}^{(\alpha)}\right)_{n}+F_{\int u}\left(u_{n}^{(\alpha)}, u_{n}, \int_{0}^{t} g\left(t, s, u_{n}(s)\right) d s, 0\right) \varepsilon \int\left(u_{c}\right)_{n} \\
& +F_{\varepsilon}\left(u_{n}^{(\alpha)}, u_{n}, \int_{0}^{t} g\left(t, s, u_{n}(s)\right) d s, 0\right) \varepsilon=0
\end{aligned}
$$

or

$$
\left(u_{c}^{(\alpha)}\right)_{n} \frac{\partial F}{\partial u^{(\alpha)}}+\left(u_{c}\right)_{n} \frac{\partial F}{\partial u}+\left(\int\left(u_{c}\right)_{n}\right) \frac{\partial F}{\partial\left(\int u\right)}+\frac{\partial F}{\partial \varepsilon}+\frac{F}{\varepsilon}=0 .
$$

Here $(.)^{\prime}$ represents the derivative according to the independent variable and

$$
F_{\varepsilon}=\frac{\partial F}{\partial \varepsilon}, F_{u}=\frac{\partial F}{\partial u}, F_{u^{\prime}}=\frac{\partial F}{\partial u^{\prime}}, \ldots
$$

The derivatives in the expansion are evaluated at $\varepsilon=0$. Beginning with an initial function $u_{0}(t)$, first $\left(u_{c}\right)_{0}(t)$ is calculated by the help of (21) and then substituted into Eq.(8) to calculate $u_{1}(t)$. Iteration procedure is continued using (21) and (8) until obtaining a reasonable solution.

\section{Convergence analysis of the PIA}

In this section we give a convergence analysis of the method.

Theorem 1. PIA $(1,1)$ converges for Eq.(3) when $\left\|u_{k+1}-u_{k}\right\| \leq \varepsilon^{\prime}$ and $\varepsilon^{\prime} \rightarrow 0$. 
Proof. The general iteration formula of $\operatorname{PIA}(m, n)$ is converted to $\operatorname{PIA}(1,1)$ in recursive relation $(21)$ by substituting $m=1$ and $n=1$ that can be stated as follows:

$$
u_{k}^{\prime}(t)+\frac{F_{u_{k}}}{F_{u_{k}^{\prime}}} u_{k}(t)=-\frac{F_{\varepsilon}+\frac{F}{\varepsilon}}{F_{u_{k}^{\prime}}} .
$$

By changing $k$ to $k+1$ in Eq.(22) to obtain a relation with respect to $u_{k+1}^{\prime}(t)$ and $u_{k}(t)$ and imposing norm 2 on both sides of equations, we have:

$$
\begin{aligned}
\left\|u_{k}^{\prime}\right\| & \leq\left\|\frac{F_{\varepsilon}+\frac{F}{\varepsilon}}{F_{u_{k}^{\prime}}}\right\|+\left\|\frac{F_{u_{k}}}{F_{u_{k}}}\right\| \cdot\left\|u_{k}\right\|, \\
\left\|u_{k+1}^{\prime}\right\| & \leq\left\|\frac{F_{\varepsilon}+\frac{F}{\varepsilon}}{F_{u_{k+1}^{\prime}}}\right\|+\left\|\frac{F_{u_{k+1}}}{F_{u_{k+1}^{\prime}}}\right\| \cdot\left\|u_{k+1}\right\| .
\end{aligned}
$$

Now, we need to obtain $\left\|u_{k+1}-u_{k}\right\|$ from $\left\|u_{k+1}^{\prime}-u_{k}^{\prime}\right\|$. By rewriting inequalities with respect to $\left\|u_{k}\right\|$ and $\left\|u_{k+1}\right\|$ and by using the magnitude rules in calculus, we get:

$$
\left\|u_{k+1}-u_{k}\right\| \geq\left\|u_{k+1}\right\|-\left\|u_{k}\right\|=\left\|\frac{F_{u_{k+1}^{\prime}}}{F_{u_{k+1}}}\right\| \cdot\left(\left\|u_{k+1}^{\prime}\right\|-\left\|\frac{F_{\varepsilon}+\frac{F}{\varepsilon}}{F_{u_{k+1}^{\prime}}}\right\|\right)-\left\|\frac{F_{u_{k}^{\prime}}^{\prime}}{F_{u_{k}}}\right\| \cdot\left(\left\|u_{k}^{\prime}\right\|-\left\|\frac{F_{\varepsilon}+\frac{F}{\varepsilon}}{F_{u_{k}^{\prime}}}\right\|\right) \text {. }
$$

So we need to obtain bound for $\left\|u_{k+1}-u_{k}\right\|$. As a result, we can find out that $\left\{u_{n}\right\}$ is a Cauchy sequence in Banach space, then it is convergent. It holds due to this fact that $f \in C^{m}[a, b]$ and $f^{m} \in C_{\mu}^{m}$ according to main definition of Banach space in real and functional analysis. All elements of $\left\|u_{k+1}-u_{k}\right\|$ in right hand side of inequality are known except $\left\|u_{k}^{\prime}\right\|$ and $\left\|u_{k+1}^{\prime}\right\|$.

$$
\left\|u_{k+1}-u_{k}\right\| \geq\left\|\frac{F_{u_{k+1}^{\prime}}}{F_{u_{k+1}}}\right\| \cdot\left\|u_{k+1}^{\prime}\right\|-\left\|\frac{F_{u_{k+1}^{\prime}}}{F_{u_{k+1}}}\right\| \cdot\left\|\frac{F_{\varepsilon}+\frac{F}{\varepsilon}}{F_{u_{k+1}^{\prime}}}\right\|-\left\|\frac{F_{u_{k}^{\prime}}}{F_{u_{k}}}\right\| \cdot\left\|u_{k}^{\prime}\right\|+\left\|\frac{F_{u_{k}^{\prime}}}{F_{u_{k}}}\right\| \cdot\left\|\frac{F_{\varepsilon}+\frac{F}{\varepsilon}}{F_{u_{k}^{\prime}}}\right\| .
$$

Since $F_{u_{k+1}^{\prime}}, F_{u_{k+1}}, F_{u_{k}}$ and $F_{u_{k}^{\prime}} \in C_{\mu}^{m}, \mu \geq-1$, then they are bounded and we have:

$$
\begin{gathered}
\left\|\frac{F_{u_{k+1}^{\prime}}}{F_{u_{k+1}}}\right\| \leq M_{1},\left\|\frac{F_{u_{k}^{\prime}}}{F_{u_{k}}}\right\| \leq M_{2}, \\
\left\|\frac{F_{\varepsilon}+\frac{F}{\varepsilon}}{F_{u_{k+1}^{\prime}}}\right\| \leq M_{1}^{\prime},\left\|\frac{F_{\varepsilon}+\frac{F}{\varepsilon}}{F_{u_{k}^{\prime}}}\right\| \leq M_{2}^{\prime} .
\end{gathered}
$$

Therefore, we obtain

$$
\left\|u_{k+1}-u_{k}\right\| \geq M_{1}\left\|u_{k+1}^{\prime}\right\|-M_{1} \cdot M_{1}^{\prime}-M_{2} \cdot\left\|u_{k}^{\prime}\right\|+M_{2} \cdot M_{2}^{\prime}
$$

Now we consider.

$$
\begin{aligned}
u_{k}^{\prime} & =L\left[u_{k}\right] \\
u_{k+1}^{\prime} & =L\left[u_{k+1}\right],
\end{aligned}
$$

where $L$ is a linear operator that is defined as $L=\frac{d}{d()}$ []. Since any linear operator is bounded in theory of operators from pure mathematics, then, we can define

$$
\left\|u_{k}^{\prime}\right\|=\left\|L\left[u_{k}\right]\right\| \leq N_{1}\left\|u_{k+1}^{\prime}\right\|=\left\|L\left[u_{k+1}\right]\right\| \leq N_{2}
$$


Therefore, we get

$$
\left\|u_{k+1}-u_{k}\right\| \geq M_{1} \cdot N_{2}-M_{1} \cdot M_{1}^{\prime}-M_{2} \cdot N_{1}+M_{2} \cdot M_{2}^{\prime}=M_{1}\left(N_{2}-M_{1}^{\prime}\right)+M_{2}\left(M_{2}^{\prime}-N_{1}\right) .
$$

If $\left\|u_{k+1}-u_{k}\right\| \rightarrow 0$ then, we have

$$
\lim _{\varepsilon^{\prime} \rightarrow 0}\left(M_{1}\left(N_{2}-M_{1}^{\prime}\right)+M_{2}\left(M_{2}^{\prime}-N_{1}\right)\right)=0
$$

If $M_{1}\left(N_{2}-M_{1}^{\prime}\right)=0$ then $M_{1}=0$ or and if $M_{2}\left(M_{2}^{\prime}-N_{1}\right)=0$ then $M_{2}=0$ or $M_{2}^{\prime}=N_{1}$. The proof is complete. The proof can be done in similar manner for $\operatorname{PIA}(1,2), P I A(2,2)$ and so on. Therefore, we can find such these conditions for PIA(1,2), PIA(2,2) states and so on. In fact, we have found the condition of stop process for PIA method by all involved expressions in the iteration algorithm. Therefore, all the governing conditions needs to be imposed on PIA method to become convergent just in a few of computational iterations.

\section{Applications}

Example 1. Consider the following nonlinear fractional Fredholm integro-differential equation [17].

$$
\frac{d^{\alpha} u(t)}{d t^{\alpha}}-\int_{0}^{1} t s u^{2}(s) d s=1-\frac{t}{4}, \quad t>0, \quad 0 \leq t<1, \quad 0<\alpha \leq 1,
$$

with the initial condition $u(0)=0$ and the known exact solution for $\alpha=1$ is

$$
u(t)=t
$$

Before iteration process rewriting Eq.(23) with adding and subtracting $u^{\prime}(t)$ to the equation gives

$$
\varepsilon \frac{d^{\alpha} u(t)}{d t^{\alpha}}-u^{\prime}(t)+\varepsilon u^{\prime}(t)-\varepsilon \int_{0}^{1} t s(u(s))^{2} d s-1+\frac{t}{4}=0 .
$$

In this case for

$$
F\left(u^{\prime}, u, \varepsilon\right)=\frac{1}{\Gamma(1-\alpha)} \varepsilon \int_{0}^{t} \frac{u^{\prime}(s)}{(t-s)^{\alpha}} d s-u_{n}^{\prime}(t)+\varepsilon u_{n}^{\prime}(t)-\varepsilon \int_{0}^{1} t s\left(u_{n}(s)\right)^{2} d s-1+\frac{t}{4},
$$

and the iteration formula

$$
u^{\prime}(t)+\frac{F_{u}}{F_{u^{\prime}}} u(t)=-\frac{F_{\varepsilon}+\frac{F}{\varepsilon}}{F_{u^{\prime}}}
$$

the terms that will be replaced in, are

$$
\begin{aligned}
F & =u_{n}^{\prime}(t)-1+\frac{t}{4} \\
F_{u} & =0 \\
F_{u^{\prime}} & =1 \\
F_{\varepsilon} & =-u_{n}^{\prime}(t)+\frac{1}{\Gamma(1-\alpha)} \int_{0}^{t} \frac{u^{\prime}(s)}{(t-s)^{\alpha}} d s-\int_{0}^{1} t s(u(s))^{2} d s .
\end{aligned}
$$


After substitution the differential equation for this problem, Eq.(21) becomes

$$
\frac{\int_{0}^{t}(-s+t)^{-\alpha} u_{n}^{\prime}(s) d s}{\Gamma(1-\alpha)}+\left(u_{c}^{\prime}(t)\right)_{n}=\int_{0}^{1} s t\left(u_{n}(s)\right)^{2} d s+\frac{4-t+4(-1+\varepsilon) u_{n}^{\prime}(t)}{4 \varepsilon}
$$

Appropriate to the initial conditions, chosen $u_{0}(t)=0$ and, solving Eq.(29) for $n=0$ gives

$$
\left(u_{c}(t)\right)_{0}=t-\frac{t^{2}}{8}+C_{1}
$$

This expression written in

$$
u_{1}=u_{0}+\varepsilon\left(u_{c}(t)\right)_{0}
$$

gives

$$
u_{1}(x, t)=u_{0}(x, t)+\varepsilon\left(t-\frac{t^{2}}{8}+C_{1}\right)
$$

or

$$
u_{1}(x, t)=\varepsilon\left(t-\frac{t^{2}}{8}+C_{1}\right)
$$

Solving this equation for

$$
u_{1}(0)=0
$$

we obtain

$$
C_{1}=0
$$

For this value and $\varepsilon=1$ reorganizing $u_{1}(t)$

$$
u_{1}(t)=t-\frac{t^{2}}{8}
$$

gives the first iteration result. If the iteration procedure is continued in a similar way, we obtain the following iterations.

$$
\begin{gathered}
u_{2}(t)=2 t-\frac{571 t^{2}}{3840}+\frac{t^{2-\alpha}(t+4(-3+\alpha))}{4 \Gamma(4-\alpha)}, \\
u_{3}(t)=3 t+\frac{29844889 t^{2}}{176947200}-\frac{t^{3-2 \alpha}(t+8(-2+\alpha))}{4 \Gamma(5-2 \alpha)} \\
+\frac{t^{2}\left(3379230+8 t^{-\alpha}(1051 t+5760(-3+\alpha))(-7+\alpha)(-6+\alpha)(-5+\alpha)\right)}{15360(-7+\alpha)(-6+\alpha)(-5+\alpha) \Gamma(4-\alpha)} \\
-\frac{2240277 \alpha+(450151-28436 \alpha) \alpha^{2}}{15360(-7+\alpha)(-6+\alpha)(-5+\alpha) \Gamma(4-\alpha)}-\frac{t^{2}(-4+\alpha)(-1159+2 \alpha(529+16(-10+\alpha) \alpha))}{64(-7+2 \alpha) \Gamma(5-\alpha)^{2}} .
\end{gathered}
$$

The other iterations contain large inputs and are not given. A computational software program could help to calculate the other iterations up to any order. In Table 1. some of the PIA iteration results are compared with the exact solutions. The results express that the present method gives highly approximate solutions. Also in Figure 1. the obtained results are illustrated graphically.

Example 2. Consider the following nonlinear Volterra type fractional integro-differential equation [6].

$$
\frac{d^{\alpha} u(t)}{d t^{\alpha}}-\int_{0}^{t} e^{-s} u^{2}(s) d s=1, \quad t>0, \quad 0 \leq t<1, \quad 0<\alpha \leq 1,
$$


Table 1: Numerical results of Example 1. for $u_{5}$ values for different values of $\alpha$.

\begin{tabular}{ccccccc}
\hline & $\alpha=0.25$ & $\alpha=0.50$ & $\alpha=0.75$ & \multicolumn{3}{c}{$\alpha=1.0$} \\
\hline$t$ & $P I A$ & $P I A$ & $P I A$ & $P I A$ & Exact Solution & Absolute Error \\
\hline 0.0 & 0.00000 & 0.00000 & 0.00000 & 0.000000 & 0.000000 & 0.000000 \\
0.1 & 0.40400 & 0.31001 & 0.19212 & 0.099981 & 0.100000 & $1.872712 \mathrm{E}-6$ \\
0.2 & 0.70027 & 0.51165 & 0.32983 & 0.199992 & 0.200000 & $7.490848 \mathrm{E}-6$ \\
0.3 & 0.92857 & 0.66680 & 0.45097 & 0.299983 & 0.300000 & $1.685440 \mathrm{E}-5$ \\
0.4 & 1.10862 & 0.79495 & 0.56312 & 0.399970 & 0.400000 & $2.996339 \mathrm{E}-5$ \\
0.5 & 1.25343 & 0.90616 & 0.66959 & 0.499953 & 0.500000 & $4.681780 \mathrm{E}-5$ \\
0.6 & 1.37247 & 1.00645 & 0.77217 & 0.599932 & 0.600000 & $6.741763 \mathrm{E}-5$ \\
0.7 & 1.47293 & 1.09971 & 0.87196 & 0.699908 & 0.700000 & $9.176289 \mathrm{E}-5$ \\
0.8 & 1.56044 & 1.18858 & 0.969682 & 0.799880 & 0.800000 & $1.198535 \mathrm{E}-4$ \\
0.9 & 1.63945 & 1.27490 & 1.06583 & 0.899848 & 0.900000 & $1.516896 \mathrm{E}-4$ \\
1.0 & 1.71351 & 1.35996 & 1.16078 & 0.999812 & 1.000000 & $1.872712 \mathrm{E}-4$ \\
\hline
\end{tabular}

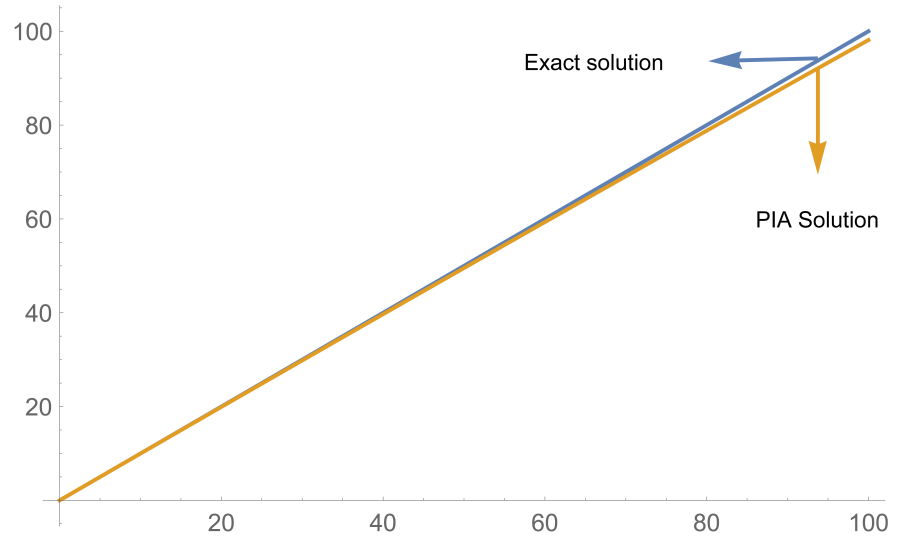

Fig. 1: Comparison of the PIA solution $u_{5}(t)$ and exact solution for Example 1. when $\alpha=1$

with the initial condition $u(0)=1$ and the known exact solution for $\alpha=1$ is

$$
u(t)=e^{t}
$$

By applying similar procedures as in the first example, we obtain the following iteration results.

$$
u_{1}(t)=1+t+\frac{t^{2}}{2}-\frac{t^{3}}{6}+\frac{t^{4}}{24}-\frac{t^{5}}{120}
$$

$$
\begin{gathered}
u_{2}(t)=1+2 t+t^{2}+\frac{t^{4}}{12}-\frac{t^{5}}{30}-\frac{t^{7}}{420}+\frac{t^{8}}{1344}-\frac{t^{9}}{576}+\frac{67 t^{10}}{86400}-\frac{217 t^{11}}{950400}+\frac{581 t^{12}}{11404800}-\frac{101 t^{13}}{13478400}+\frac{t^{14}}{1209600}-\frac{t^{15}}{18144000} \\
+\frac{t^{2-\alpha}\left(t^{4}+t^{3}(\alpha-6)+t^{2}(\alpha-6)(\alpha-5)+(t-1)((\alpha-6)(\alpha-5)(\alpha-4)-(\alpha-3))\right)}{\Gamma(7-\alpha)}
\end{gathered}
$$

and so on. The third iteration result $\left(u_{3}\right)$ is calculated in this manner. In Table 2. some of the PIA iteration results are compared with the results from Laplace variational iteration method, and exact solutions. The results express that the present method gives highly approximate solutions. Also , in Figure 2. the obtained results are illustrated graphically.

\section{Conclusion}

In this study, Perturbation-Iteration Algorithm was introduced for some Fredholm and Volterra type fractional-integro differential equations and the convergence analysis of the method is given for the first time. The application and results 
Table 2: Numerical results of Example 2. for $u_{3}$ values for different values of $\alpha$.

\begin{tabular}{ccccccccc}
\hline & $\alpha=0.25$ & $\alpha=0.50$ & $\alpha=0.75$ & & & & $\alpha=1.0$ & \\
\hline $\mathrm{t}$ & $P I A$ & $P I A$ & $P I A$ & LVIM & $P I A$ & Exact & Absolute Error LVIM & Absolute Error PIA \\
\hline 0.0 & 1.00000 & 1.00000 & 1.00000 & 1.000000 & 1.00000 & 1.00000 & 0.000000 & 0.000000 \\
0.1 & 1.28212 & 1.24644 & 1.18464 & 1.11051 & 1.10517 & 1.10517 & $7.51413 \mathrm{E}-6$ & $1.73951 \mathrm{E}-9$ \\
0.2 & 1.55024 & 1.46729 & 1.35031 & 1.22151 & 1.22140 & 1.22140 & $1.07525 \mathrm{E}-4$ & $1.35655 \mathrm{E}-7$ \\
0.3 & 2.81414 & 1.68260 & 1.51800 & 1.35034 & 1.34986 & 1.34986 & $4.81654 \mathrm{E}-4$ & $1.84719 \mathrm{E}-6$ \\
0.4 & 2.07933 & 1.90032 & 1.69403 & 1.49315 & 1.49181 & 1.49182 & $1.32812 \mathrm{E}-3$ & $1.22371 \mathrm{E}-5$ \\
0.5 & 2.35017 & 2.12576 & 1.88236 & 1.65149 & 1.64867 & 1.64872 & $2.77549 \mathrm{E}-3$ & $5.44797 \mathrm{E}-5$ \\
0.6 & 2.63047 & 2.36307 & 2.08602 & 1.82691 & 1.82193 & 1.82212 & $4.79581 \mathrm{E}-3$. & $1.88391 \mathrm{E}-4$ \\
0.7 & 2.92353 & 2.61564 & 2.30752 & 2.02086 & 2.01321 & 2.01375 & $7.11310 \mathrm{E}-3$ & $5.46883 \mathrm{E}-4$ \\
0.8 & 3.23214 & 2.88627 & 2.54898 & 2.23464 & 2.22414 & 2.22554 & $9.10286 \mathrm{E}-3$ & $1.39627 \mathrm{E}-3$ \\
0.9 & 3.55846 & 3.17710 & 2.81216 & 2.46928 & 2.45637 & 2.45960 & $9.67744 \mathrm{E}-3$ & $3.23130 \mathrm{E}-3$ \\
1.0 & 3.90380 & 3.48951 & 3.09828 & 2.72543 & 2.71136 & 2.71828 & $7.15178 \mathrm{E}-3$ & $6.00691 \mathrm{E}-3$ \\
\hline
\end{tabular}

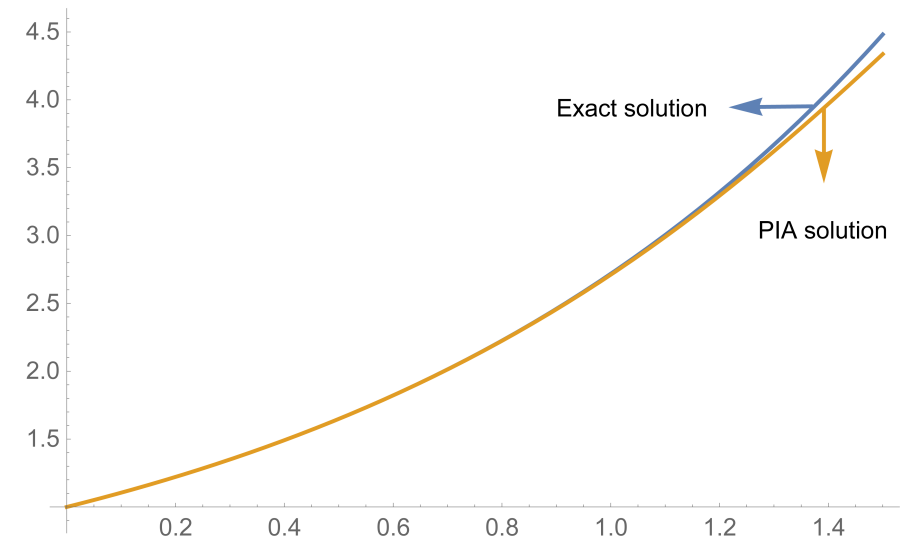

Fig. 2: Comparison of the PIA solution $u_{3}(t)$ and exact solution for Example 2. when $\alpha=1$

show that the method is very simple and reliable perturbation-iteration technique and producing highly approximate results. We expect that the present method can be used to calculate the approximate solutions of other types of fractional differential equations.

\section{Competing interests}

The authors declare that they have no competing interests.

\section{Authors' contributions}

All authors have contributed to all parts of the article. All authors read and approved the final manuscript.

\section{References}

[1] Aksoy Y. and Pakdemirli M., New perturbation-iteration solutions for Bratu-type equations, Comput Math Appl. 59 (2010), 28022808.

[2] Aksoy Y., Pakdemirli M., Abbasbandy S. and Boyaci H., New perturbation-iteration solutions for nonlinear heat transfer equations, Int J Heat Fluid Fl. 22 (2012), 814-828. 
[3] Arikoglu A. and Ozkol I., Solution of fractional integro-differential equations by using fractional differential transform method, Chaos Soliton Fract. 40 (2009), 521-529.

[4] Baskonus, H. M. and Bulut H., On the numerical solutions of some fractional ordinary differential equations by fractional AdamsBashforth-Moulton method, Open Math, 13(1) (2015), 547-556.

[5] Baskonus, H. M., Mekkaoui, T., Hammouch, Z. and Bulut, H., Active control of a chaotic fractional order economic system, Entropy, 17(8) (2015), 5771-5783.

[6] Biala T. A., Afolabi Y.O. and Asim O.O., Laplace variational iteration method for integro-differential equations of fractional order, Int J Pure Appl Math. 95.3 (2014), 413-426.

[7] Cavlak E. and Bayram M., An approximate solution of fractional cable equation by homotopy analysis method, Bound. Value Probl., 2014(1), 58.

[8] Cooper K. and Mickens R. E., Generalized harmonic balance/numerical method for determining analytical approximations to the periodic solutions of the $x^{4 / 3}$ potential, J. Sound Vibr. 250 (2002), 951-954.

[9] Dolapci İ. T., Şenol M. and Pakdemirli M., New perturbation iteration solutions for Fredholm and Volterra integral equations, J Appl Math. (2013).

[10] El-Sayed A., Nour H., Raslan W. and El-Shazly E., A study of projectile motion in a quadratic resistant medium via fractional differential transform method, Appl Math Model. 39.10 (2015), 2829-2835.

[11] Esen A. and Tasbozan O., Numerical solution of time fractional nonlinear Schrodinger equation arising in quantum mechanics by cubic B-spline finite elements, Malaya J. Mat., 3(4) (2015), 387-397.

[12] Esen A. and Tasbozan O., An approach to time fractional gas dynamics equation: Quadratic B-spline Galerkin method, Appl. Mat. Comput., 261 (2015), 330-336.

[13] G. von Groll and Ewins D.J., The harmonic balance method with arc-length continuation in rotor/stator contact problems, J. Sound Vibr. 241 (2001), 223-233.

[14] Heaviside O., Electromagnetic theory, Cosimo Inc.2008.

[15] He J. H., Iteration Perturbation Method for Strongly Nonlinear Oscillators, J. Sound Vibr. 7 (2001), 631-642.

[16] He J. H., Homotopy perturbation method with an auxiliary term, Abst Appl Anal. 2012.

[17] Hou J., Qin B. and Yang C., Numerical Solution of Nonlinear Fredholm Integrodifferential Equations of Fractional Order by Using Hybrid Functions and the Collocation Method, J Appl Math. 2012.

[18] Hu H. and Xiong Z.G., Oscillations in an $x^{(2 m+2) /(2 n+1)}$ potential, J. Sound Vibr. 259 (2003), 977-980.

[19] İbiş B. and Bayram M., Approximate solution of time-fractional advection-dispersion equation via fractional variational iteration method, The Scientific World Journal, 2014.

[20] İbiş B. and Bayram M., Numerical comparison of methods for solving fractional differential-algebraic equations (FDAEs), Comput Math Appl., 62(8) (2011), 3270-3278.

[21] Iqbal S and Javed A., Application of optimal homotopy asymptotic method for the analytic solution of singular Lane-Emden type equation, Appl Math and Comput. 217 (2011), 7753-7761.

[22] Jordan D. W. and Smith P., Nonlinear ordinary differential equations: An introduction to dynamical systems, Vol. 2, Oxford University Press, USA, (1999).

[23] Kurt A. and Tasbozan O., Approximate analytical solution of the time fractional Whitham-Broer-Kaup equation using the Homotopy Analysis Method, International Journal of Pure and Applied Mathematics, 98(4) (2015), 503-510.

[24] Luchko Y. F. and Srivastava H.M., The exact solution of certain differential equations of fractional order by using operational calculus, Comput Math Appl. 29.8 (1995), 73-85.

[25] Mainardi F., Fractals and fractional calculus in continuum mechanics, Springer Verlag, 1997.

[26] Mickens R. E., Iteration procedure for determining approximate solutions to non-linear oscillator equations, J. Sound Vibr. 116 (1987), 185-187.

[27] Mickens R. E., A generalized iteration procedure for calculating approximations to periodic solutions of truly nonlinear oscillators, J. Sound Vibr. 287 (2005), 1045-1051.

[28] Mickens R. E., Iteration method solutions for conservative and limit-cycle $x^{1 / 3}$ force oscillators, J. Sound Vibr. 292 (2006), $964-$ 968.

[29] Momani S., Odibat Z. and Erturk V. S., Generalized differential transform method for solving a space-and time-fractional diffusionwave equation, Phys Lett A. 370 (2007), 379-387. 
[30] Nayfeh A. H, Perturbation methods, John Wiley \& Sons, 2008.

[31] Oldham K. B., Fractional differential equations in electrochemistry, Adv Eng Softw. 41 (2010), 9-12.

[32] Pakdemirli M., Aksoy Y. and BoyacıH., A New Perturbation-Iteration Approach for First Order Differential Equations, Math Comput Appl. 16 (2011), 890-899.

[33] Podlubny I., Fractional differential equations: an introduction to fractional derivatives, fractional differential equations, to methods of their solution and some of their applications, Academic press, 1998.

[34] Senol B., Ates A., Alagoz B. B. and Yeroglu C., A numerical investigation for robust stability of fractional-order uncertain systems, Isa T. 53 (2014), 189-198.

[35] Şenol M., Dolapci İ. T., Aksoy Y. and Pakdemirli M., Perturbation-Iteration Method for First-Order Differential Equations and Systems, Abstr Appl Anal. 2013.

[36] Şenol M. and Dolapci İ. T., On the Perturbation-Iteration Algorithm for fractional differential equations, J King Saud Univ Sci. 28.1 (2016), 69-74.

[37] Skorokhod A. V., Hoppensteadt F.C. and Salehi H.D., Random perturbation methods with applications in science and engineering, Springer Science \& Business Media, 2002.

[38] Toyoda M. and Watanabe T., Existence and uniqueness theorem for fractional order differential equations with boundary conditions and two fractional order, J. Nonlinear Convex Anal. 17.2 (2016), 267-273.

[39] Wang S. Q. and He J. H., Nonlinear oscillator with discontinuity by parameter-expansion method, Chaos Soliton Fract. 35 (2008), 688-691.

[40] Yakar A. and Koksal M. E., Existence results for solutions of nonlinear fractional differential equations, Abstr Appl Anal. 2012.

[41] Yu Z. S. and Lin J. Z., Numerical research on the coherent structure in the viscoelastic second-order mixing layers, Appl Math Mech-Engl. 19 (1998), 717-723. 